\title{
Wind Turbine System based Virtual Synchronous Generator Control for Microgrid Frequency Regulation
}

\author{
Haseeb Ur Rehman ${ }^{1 *}$, Xiangwu Yan ${ }^{1}$, Mishkat Ullah Jan ${ }^{2 *}$, Mohamed Abdelkarim Abdelbaky ${ }^{2}$, Sheeraz Iqbal ${ }^{2}$, Tamara \\ Egamnazarova $^{2}$, Syed Asad Abbas Ali Rizvi² \\ ${ }^{1}$ School of Electrical \& Electronic Engineering, North China Electric Power University, Baoding, 071003, China. \\ ${ }^{2}$ State Key Laboratory of Alternate Electrical Power System with Renewable Energy Source (NCEPU), Beijing 102206, China.
}

\begin{abstract}
The power system is dominated by the renewable energy resources since last two decades. The main cause behind this enormous deployment of RES is to combat the global warming and to decrease dependency on fossil fuels. Wind energy is one of the abundant available sources of energy throughout the world. However, the huge deployment of wind energy in the power system cause vulnerability to the system. The main cause is the lack of system inertia in RES which cannot effectively respond during the power imbalance condition. To counter this effect, the virtual synchronous generators are deployed in the RES based power system. The VSG mimics the synchronous generator features and therefore it possesses the ability to provide the inertial support to system. In this paper, a wind base multiple VSG system is proposed to counter the frequency irregularities of the system. Furthermore, a comparison with classical droop control is done based on load variation. Moreover, the genetic algorithm is used to optimize the values of damping and inertia of the VSG. The performance of the proposed system is validated in MATLAB/Simulink 2019.
\end{abstract}

\section{Introduction}

The recent ecological changes and natural disasters (i.e. flood and pandemic) around the globe and the rapid growth of population, has forced the human kind to discover new resources for power generation. The COVID-19 has affected the world's economy and development. Most of businesses have gone online due to immobility, which requires a huge amount of power to cope with the user demand. The renewable energy sources (RESs) has played a vital role to cater with the power deficit. As on 2020, China has the wind power capacity of $281.5 \mathrm{GW}$, which is predicted to reach the target of 400 GW by 2030 [1]. According to the Paris Accord, the largest economies are compelled to produce/generate more clean and green power, in order to decrease the global warming issues. For this reason, China has been transforming its electric power generation from fossil fuel-based power plants to renewable generation. The total RESs generated by China since 2019 was $758 \mathrm{GW}$. To integrate RESs into power system a huge technological advancement is the cry of the day. The integration of RESs into the power system causes various problems like frequency and voltage instability.

The common RESs explored throughout the world are solar and wind besides hydro power. The first two are abundantly available throughout the world. However, the hydro power depends upon the water channel. Therefore, wind and solar are commonly attributed as RESs. The main problem in the RESs is the lack of inertia. So, they cannot contribute to the system power imbalance. The system inertia is defined as the ability of the system at which it responds to the variations in load.

Various researchers proposed different control techniques to restore the inertial problem in RESs. Frequency regulation of an isolated $\mathrm{MG}$ is studied using genetic optimization technique [2, 3]. Similarly, in [4] the primary frequency regulation of a grid connected RES is studied. Moreover, a multi-VSG system with PV is studied in [5,6]. Additionally, the parameters of VSG are well optimized. VISMA [7] and VSG [8] are incorporated in such way that the grid assumes them as a synchronous generator.

There are two modes of virtual synchronous generator (VSG) used in the literature. The first one is voltage source type which can operate stably in the islanded power networks without phase locked loop (PLL). Meanwhile, the current source type is deployed in the grid connected network. Moreover, in current source type VSG the PLL is an important parameter which should be considered for accurate calculation [9]. The wind turbine system possesses less amount of system inertia [10,11]. The above authors either didn't considered the WTS or the wind control parameters optimization for this reason a wind-based VSG scheme is studied in this paper. The internal characteristics of wind turbine system are ignored in order to fully exploit the performance of VSG.

This paper is divided into five parts. Part II includes the dynamic model of wind-based VSG system. Part III includes problem formulation and optimization 
techniques. The simulation and results are presented in part IV, and finally conclusion is drawn in part V.

\section{System Modelling}

The power grid consists of generators and the load where the swing equation provides the complete details of power system. Equation (1) represents the swing equation.

$$
J \frac{\partial\left(\omega-\omega_{g}\right)}{\partial t}=\frac{P_{r e f}-P}{\omega}-D\left(\omega_{g}-\omega\right)
$$

\subsection{Wind Turbine System}

The wind turbine system and its variants are deployed in power grid to generate electric power. It is due to the fact that wind energy is abundantly available throughout the world. However, the speed of wind varies from area to area. For this reason, wind speed is one of the important parameters for designing a wind turbine system. The output power of wind turbine system is calculated from equation (2) to (4) $[12,13]$.

$$
\begin{gathered}
P_{\text {wind }}=\frac{1}{2} C_{p}(\lambda, \beta) A \rho_{a} V^{3} \\
C_{p}=0.5176\left(\frac{116}{\lambda_{i}}-0.4 \beta-5\right) e^{\frac{-21}{\lambda_{i}}}+0.0068 \lambda \\
\frac{1}{\lambda_{i}}=\frac{1}{\lambda+0.08 \beta}-\frac{0.035}{\beta^{3}+1}
\end{gathered}
$$

Where $\rho$ and $\eta$ stands for density of air and betz's constant, respectively; the tip speed ratio is given by $\lambda=$ $\frac{\omega_{r} R}{v}, \omega_{r}$ stands for rotor's angular velocity while blade length is denoted by $R$. The output power is variable due to the fact that wind speed (V) and its direction is never constant. From the literature it is evident that resultant power of WTS is a combination of coefficient of power $C_{p}$ and some other natural components. In general, $C_{p}$ is a function of two essential entities that is angle of blade pitch $(\beta)$ and the tip speed ratio $(\lambda)$. The wind turbine system is connected through a VSG to the power system. Moreover, the inertial characteristics of wind turbine system are neglected in this study.

\subsection{Virtual Synchronous Generator}

The damping and inertia of RESs are low, thus for its integration the stability and dynamic features of the electric grid ought to be enhanced. For this purpose, the power grid is provided by virtual inertia through battery storage and suitable control mechanism, which can be depicted from Figure. 2. VSG is defined as a control scheme based on inverters that bears the features of an SG. In general, the inertia and damping factor for a specified SG are considered constant [14]. However, the mentioned parameter are the vital entities that efficiently regulate the dynamic features of VSG as per system demand. Consequently, the primary emphasis has been given to enhance the dynamic attributes of the VSG in this paper. Moreover, the active and reactive power control can be seen in Figure (1).

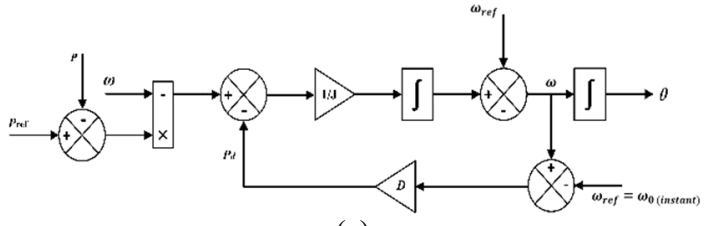

(a)

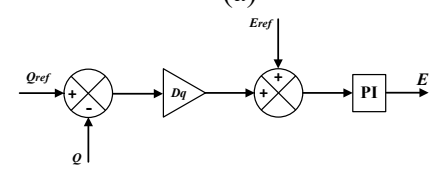

(b)

Figure 1. The control configuration of VSG (a) Active power control (b) Reactive power-voltage control

\section{Problem Formulation and Optimization}

\subsection{Problem Formulation}

Wind turbine systems (WTS) are integrated into power grid, however the WTS possess less inertia. To counter this problem a VSG system is incorporated. The VSG imitate the characteristics of synchronous generator and provides the inertia by effectively using the battery storage system (BSS) and a proper control mechanism. In this paper an advanced optimization technique named genetic algorithm (GA) is utilized.

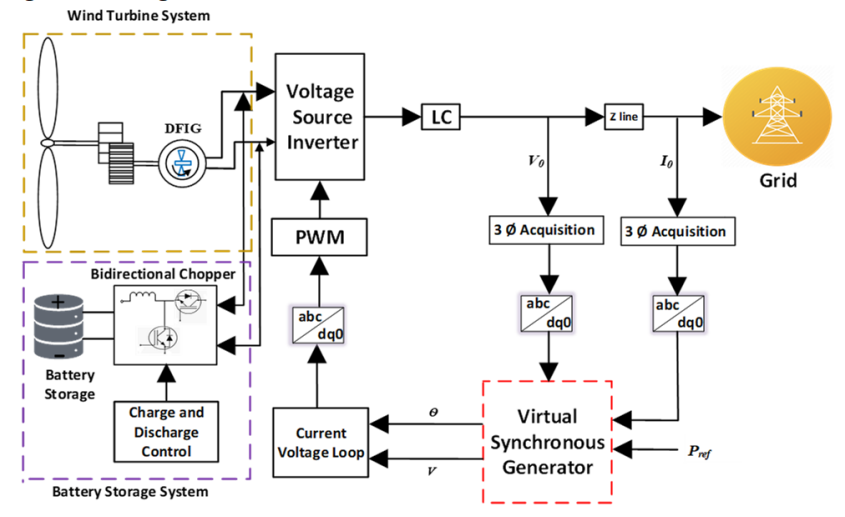

Figure 2. The proposed structure of VSG.

\subsection{Genetic Algorithm}

It is an optimization method that can execute quick hunts in a huge quantity of vague or incomplete informational collection with a built-in structure that allow parallelization. It can incorporate into the constraints of the fitness function, execution records, and purpose which is not basically all around characterized positively. Additionally, more limitations are added to express a potential entity as a fit answer for the given problems is definitely not a computational weight. In the recent research, GA has been utilized to cope with these issues in various fields. Their fame continues to increment because of their usability, adequacy, or relevance.

To execute the evolutionary algorithm (EA), a sequence of stages is tailed as: (1) the solution population and it's fitness evaluation function are initialized; (2) 
some individuals are selected and recombined to create offspring; as long as a formerly stated termination condition is not met, for each generation, based on their computed fitness, and (3) the existing population fitness is assessed to be utilized in the subsequent cycle.

Fitness $=\min \left(0.001 *\left(\operatorname{sum}\left(f_{V S G 1}\right)+\operatorname{sum}\left(f_{V S G 2}\right)+\operatorname{sum}\left(f_{V S G 3}\right)\right)+0.999 *\right.$ $\left.\left(\operatorname{sum}\left(\Delta f_{V S G 1}\right)+\operatorname{sum}\left(\Delta f_{V S G 2}\right)+\operatorname{sum}\left(\Delta f_{V S G 3}\right)\right)\right)$

\section{Simulation and Results}

The system's parameters for the multiple VSG system are given in Table 1. For optimum values, in Table 2 parameters of genetic algorithm are illustrated, while Eq. (5) depicts the fitness function.

Table 1. Multi-VSG parameters for the Micro grid system.

\begin{tabular}{|c|c|c|c|}
\hline Parameter/Value & VSG-1 & VSG-2 & VSG-3 \\
\hline$P$ & $15 \mathrm{~kW}$ & $15 \mathrm{~kW}$ & $15 \mathrm{~kW}$ \\
\hline$V_{r e f}$ & $380 \mathrm{~V}$ & $380 \mathrm{~V}$ & $380 \mathrm{v}$ \\
\hline$\omega$ & $2 \pi \times 50$ & $2 \pi \times 50$ & $2 \pi \times 50$ \\
\hline$f_{n}$ & $50 \mathrm{~Hz}$ & $50 \mathrm{~Hz}$ & $50 \mathrm{~Hz}$ \\
\hline$R_{1}$ & 0.05 & 0.05 & 0.05 \\
\hline$L_{1}$ & $1.45 \times 10-$ & $1.45 \times 10-3$ & $1.45 \times 10-3$ \\
& 3 & & \\
\hline$C_{1}$ & $150 \times 10-6$ & $150 \times 10-6$ & $150 \times 10-6$ \\
\hline$V_{d c}$ & $800 \mathrm{~V}$ & $800 \mathrm{~V}$ & $800 \mathrm{~V}$ \\
\hline
\end{tabular}

Table 2. Genetic Algorithm's parameters.

\begin{tabular}{|c|c|}
\hline Parameter & Value \\
\hline Variables' Number & 5 \\
\hline Variables & $D, J, D_{q}, K_{p}, K_{i}$ \\
\hline Lower Bounds & $\left.\begin{array}{lllll}0.001 & 0.001 & 0.001 & 0.001 & 0.001\end{array}\right]$ \\
\hline Upper Bounds & {$\left[\begin{array}{lllll}100 & 10 & 10 & 100 & 10\end{array}\right]$} \\
\hline Mutation & $80 \%$ \\
\hline Iterations & 50 \\
\hline
\end{tabular}

\subsection{Case 1: Unit Step Change in Load Power Using Advanced Droop Control.}

The first case is based on the step change in load power as illustrated in Fig. 3a. The optimum values are deduced using GA for the wind based multiple VSG system that can be depicted from Table 3. The performance and comparison of ADC with classical droop controller is illustrated in Fig. 3.

Table 3. Optimized advanced droop control parameters.

\begin{tabular}{|c|c|c|c|}
\hline Parameter/Values & VSG-1 & VSG-2 & VSG-3 \\
\hline$D_{V S G}$ & 83.8305 & 83.8305 & 83.8305 \\
\hline$D_{q}$ & 3.89895 & 3.89895 & 3.89895 \\
\hline$J$ & 9.79005 & 9.79005 & 9.79005 \\
\hline
\end{tabular}

The mean power delivered to the micro grid is steadied shortly at $\mathrm{t}=5.3 \mathrm{~s}$ and remains constant as in Fig. $3 \mathrm{~b}$. The angular frequency and system frequency are maintained within the nominal range and are continuously kept constant by the VSG control as in Fig 3c-3d.
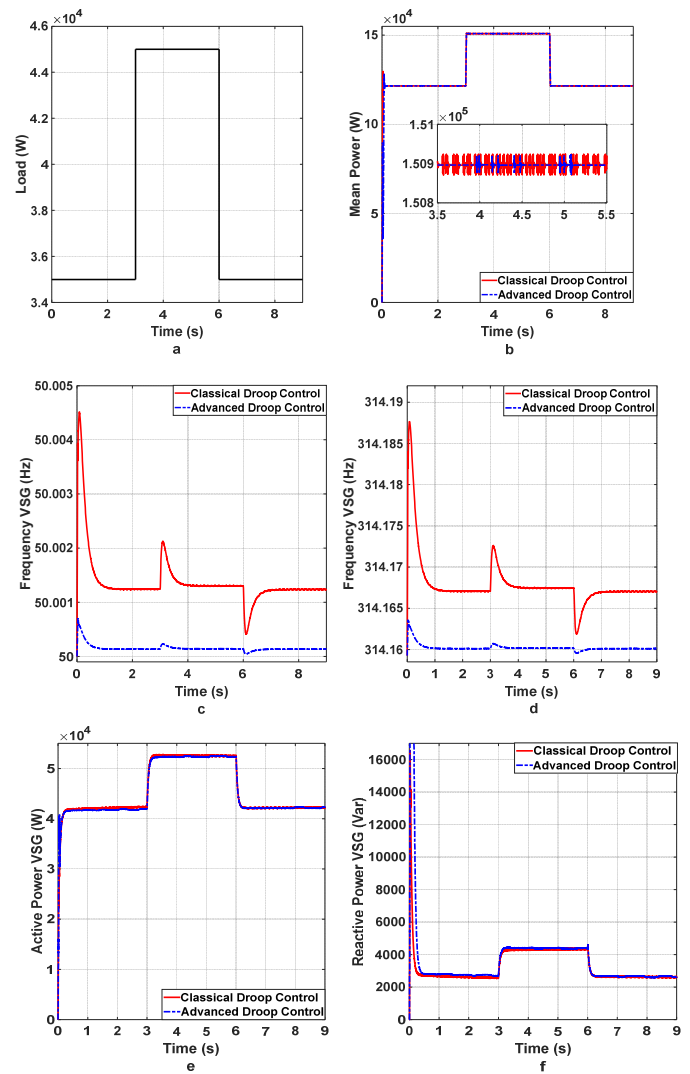

Fig. 3. Comparisons of the classical and advanced droop control: (a) load (b) mean power (c) frequency (d) angular frequency (e) active power (f) reactive power.

The initial variations at $\mathrm{t}=0$ in the frequency is because of the WTS integration into the micro grid. From Fig. 3e-3f, it can be seen that the active and reactive power of the multiple VSG are steadied and remain constant. It is evident from the results that the ADC performs better than the conventional/classical droop control in minimizing the overshoot and settling time of the system.

Table 4. Optimized advanced droop control parameters.

\begin{tabular}{|c|c|c|c|}
\hline Parameter/Values & VSG-1 & VSG-2 & VSG-3 \\
\hline$D_{V S G}$ & 28.7605 & 28.7605 & 28.7605 \\
\hline$D_{q}$ & 9.5418 & 9.5418 & 9.5418 \\
\hline$J$ & 0.9006 & 0.9006 & 0.9006 \\
\hline
\end{tabular}

\subsection{Case 2: Large Step Change in Load Power Using Droop Control.}

This case is based on huge step change in the load power as compare to the first case which can be depicted from Fig. 4a. The optimum values are deduced using GA for the wind based multiple VSG system that can be depicted from Table 4. The performance and comparison of ADC with classical droop controller is illustrated in Fig. 4. 

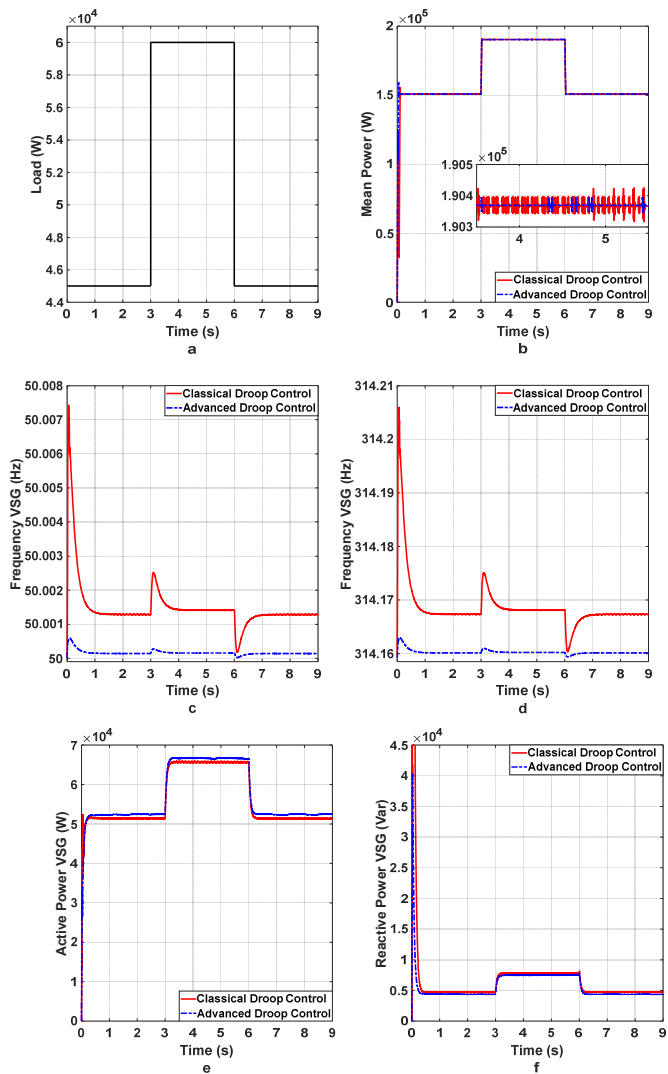

Fig. 4. Comparisons of the classical and advanced droop control: (a) load (b) mean power (c) frequency (d) angular frequency (e) active power (f) reactive power

The mean power delivered to the micro grid is steadied shortly at $\mathrm{t}=5.7 \mathrm{~s}$ and remains steady as in Fig. $4 \mathrm{~b}$. The angular frequency and system frequency are maintained at the nominal values within the range as in Fig 4c-4d. From Fig. 4e-4f, it can be seen that the active and reactive power of the multiple VSG are steadied and remain constant by the VSG. Moreover, it is also evident from the results that the ADC performs better than the conventional/classical droop control in minimizing the overshoot and settling time of the system.

\section{Conclusion}

In this paper a wind based multiple VSG system is studied to analyze the frequency stability of the system. The frequency stability of a micro grid and its reaction to load variation is observed via WTS based multiple VSG system. Moreover, for optimum values of rotational inertia, droop coefficient and damping, an advanced GA is utilized in every case. Furthermore, a comparison with classical droop control is done based on load variation. The optimum values of the control parameters guarantee the stability of reactive and active power of the multi-VSG system during a short interval. The simulation results show better performance over classical droop control in minimizing the overshoot and settling time of the system.

\section{Acknowledgment}

This research is funded by National Natural Science Foundation of China-Hebei on the project of "Research on inertia characteristics and control strategy for primary frequency and voltage regulation of DFIG" (No. E201802134).

\section{References}

1. Q. Ji and D. Zhang, "How much does financial development contribute to renewable energy growth and upgrading of energy structure in China?," Energy Policy, vol. 128, pp. 114-124, 2019.

2. M. U. Jan, A. Xin, M. A. Abdelbaky, H. U. Rehman, and S. Iqbal, "Adaptive and fuzzy PI controllers design for frequency regulation of isolated microgrid integrated with electric vehicles," IEEE Access, vol. 8, pp. 87621-87632, 2020.

3. M. U. Jan, A. Xin, H. U. Rehman, M. A. Abdelbaky, S. Iqbal, and M. Aurangzeb, "Frequency Regulation of an Isolated Microgrid With Electric Vehicles and Energy Storage System Integration Using Adaptive and Model Predictive Controllers," IEEE Access, vol. 9, pp. 14958-14970, 2021.

4. S. Iqbal et al., "Aggregation of EVs for Primary Frequency Control of an Industrial Microgrid by Implementing Grid Regulation \& Charger Controller," IEEE Access, vol. 8, pp. 141977-141989, 2020.

5. H. U. Rehman, X. Yan, M. A. Abdelbaky, M. U. Jan, and S. Iqbal, "An advanced virtual synchronous generator control technique for frequency regulation of grid-connected PV system," International Journal of Electrical Power \& Energy Systems, vol. 125, p. 106440, 2021.

6. H. U. Rehman, X. Yan, M. A. Abdelbaky, M. U. Jan, S. Iqbal, A. Masood, T. Egamnazrova and $\mathrm{M}$. Aurangzeb, "Droop Control Design Based on Advanced Particle Swarm Optimization for GridConnected Multi PV-VSG," in 16th IET International Conference on AC and DC Power Transmission, 2020.

7. X. Yan and W. Zhang, "Review of VSG controlenabled universal compatibility architecture for future power systems with high-penetration renewable generation," Applied Sciences, vol. 9, no. 7, p. 1484, 2019.

8. X. Yan, A. Rasool, F. Abbas, H. Rasool, and H. Guo, "Analysis and optimization of the coordinated multivsg sources," Electronics, vol. 8, no. 1, p. 28, 2019.

9. X. Yan et al., "A novel two-stage photovoltaic gridconnected inverter voltage-type control method with failure zone characteristics," Energies, vol. 11, no. 7, p. $1865,2018$.

10. H. Shao et al., "Equivalent modeling and comprehensive evaluation of inertia emulation control strategy for DFIG wind turbine generator," IEEE Access, vol. 7, pp. 64798-64811, 2019. 
11. W. Xiaosheng, S. Peng, S. Dawei, and L. Hui, "Frequency Regulation Characteristics Optimization and Analysis of DFIG-VSG Based on Rotor Inertia Control," in 2018 China International Conference on Electricity Distribution (CICED), 2018.

12. M. A. Abdelbaky, X. Liu, and X. Kong, "Wind turbines pitch controller using constrained fuzzyreceding horizon control," in 2019 Chinese Control And Decision Conference (CCDC), 2019, pp. 236241: IEEE.
13. S. Ali, G. Yang, and C. Huang, "Performance optimization of linear active disturbance rejection control approach by modified bat inspired algorithm for single area load frequency control concerning high wind power penetration," ISA transactions, vol. 81, pp. 163-176, 2018.

14. X. Yan et al., "Adaptive-MPPT-based control of improved photovoltaic virtual synchronous generators," Energies, vol. 11, no. 7, p. 1834, 2018. 